\title{
Serum free culture and characterization of human embryonic germ cells
}

\author{
JinLian Hua ${ }^{1, *}$, Hai-sheng Yu ${ }^{1}$, Sheng Liu ${ }^{1}$, Zhongying Dou ${ }^{1, *}$ \\ ${ }^{1}$ Shaanxi Centre of Stem Cells Engineering \& Technology, Northwest A\&F University, Yangling, Shaanxi, 712100, China
}

Many proteins and growth factors in serum play important roles in the propagation of embryonic stem (ES) cells. But there are some disadvantages in using the serum to culture ES cells. We examined the effects of a chemicallydefined culture system knock-out serum replacement (KSR) in the absence of FBS on the growth and differentiation of human primordial germ cells (PGCs) and embryonic germ (EG) cells. PGCs collected from gonadal ridges and mesenteries of 4 13 week fetuses, were cultured on mouse embryonic fibroblast (MEF) or STO feeder layers. PGCs in KSR-supplemented medium proliferated, maintaining round and typical stem celllike morphologies. In addition, the efficiency of EG cell lines in KSR medium were significantly higher than that cultured in FCS medium $(P<0.05)$. The percentage of undifferentiated colonies of EG cells was significantly higher in KSR medium than in FBS-based medium $(P<\mathbf{0 . 0 5})$. The cultured cells have been continuously maintained undifferentiated state for 14 passages on MEF or STO feeder layer with serum-free culture medium. These results suggest that KSR can be used for sustaining an undifferentiated state of PGCs and EG cells in vitro. The human embryonic germ cells(hEGCs) colonies were typically like mouse EG colonies in morphology, tight, compact, multilayer cells with large and prominent nuclei, and showed normal and stable diploid karyotype, expressing AP, SSEAs and glycoprotein markers such as SSEA-1, SSEA-3, SSEA-4,TRA-1-60, TRA-1-81, and Oct-4, PCNA, C-kit, and with high telomerase activity. In contrast, mouse EGCs do not express SSEA-3, SSEA-4, TRA-1-60,TRA-1-81. Human EG cells can form simple and cystic embryoid bodies (EBs) that consist various cell types including all three embryonic germ layers, such as neural cells, epithelia-like, fibroblast-like, polygon cells and rhythmically beating myocardium-like cells ,even sperm and oocytelike cells. These germ like cells are positive for Oct4,C-kit and Scp3. So we built a appropriate serum-free culture system for the establishment of human EG cell lines and obtained pluripotent human EG cell lines derived from PGCs.

Keywords: human, primordial germ cell (PGCs), embryonic germ cells (EGCs), serum-free, pluripotent

Cell Research (2008) 18:s41. doi: 10.1038/cr.2008.131; published online 4 August 2008

Correspondence: Zhongying Dou

E-mail: jlhua2003@126.com,douzhongying@china.com 